University of Nebraska - Lincoln

DigitalCommons@University of Nebraska - Lincoln

Papers in the Earth and Atmospheric Sciences Earth and Atmospheric Sciences, Department

4-1996

\title{
Theory of Dipole Flow in Uniform Anisotropic Aquifers
}

Vitaly A. Zlotnik

University of Nebraska-Lincoln, vzlotnik1@unl.edu

Glenn Ledder

University of Nebraska-Lincoln, gledder1@unl.edu

Follow this and additional works at: https://digitalcommons.unl.edu/geosciencefacpub

Part of the Earth Sciences Commons

Zlotnik, Vitaly A. and Ledder, Glenn, "Theory of Dipole Flow in Uniform Anisotropic Aquifers" (1996).

Papers in the Earth and Atmospheric Sciences. 161.

https://digitalcommons.unl.edu/geosciencefacpub/161

This Article is brought to you for free and open access by the Earth and Atmospheric Sciences, Department of at DigitalCommons@University of Nebraska - Lincoln. It has been accepted for inclusion in Papers in the Earth and Atmospheric Sciences by an authorized administrator of DigitalCommons@University of Nebraska - Lincoln. 


\title{
Theory of dipole flow in uniform anisotropic aquifers
}

\author{
Vitaly Zlotnik \\ Department of Geology, University of Nebraska, Lincoln \\ Glenn Ledder \\ Department of Mathematics, University of Nebraska, Lincoln
}

\begin{abstract}
A theory of dipole flow is developed to model flow induced by a vertical circulation well consisting of injection and extraction chambers in a single borehole. Included in the theory are an analytical description of the kinematic flow structure around a vertical circulation well and the drawdown in the well chambers. Using Stokes' stream function, simple criteria are derived to determine the region of intensive recirculation. This region extends (from the dipole center) approximately five distances between chamber centers in the radial direction and two distances between chamber centers in both vertical directions. The vertical scale does not depend on anisotropy of hydraulic conductivity, and the radial scale is corrected for anisotropic aquifers. Based on these estimates, criteria are given for the selection of the appropriate aquifer model to employ in five settings, including infinite, semi-infinite confined, semi-infinite unconfined, finite confined, and finite unconfined aquifers. Applications of dipole flow theory are given for analytical estimation of the capture zone for recirculation wells and for simultaneous measurement of horizontal and vertical hydraulic conductivity in uniform anisotropic aquifers using steady state measurements of drawdown in the well chambers.
\end{abstract}

\section{Introduction}

During the last few years, analysis of flow around recirculation wells has become an important issue in subsurface hydrology [Herrling et al., 1991; Gvirtzman and Gorelick, 1992; Kabala, 1993; Falta, 1995]. A vertical circulation well is a vertical well consisting of two screened sections separated by an impermeable casing, with a small pump circulating water between the upper and lower chambers. These devices induce a flow characterized by no net withdrawal of water from the aquifer; in the absence of ambient flow, streamlines proceed from the injection chamber through the aquifer to the extraction chamber. Other types of recirculation wells are being explored [Gvirtzman and Gorelick, 1992; Falta, 1995].

Flow induced by a combination of a point source (water injection) and a point sink (water extraction) is well known in hydrodynamics as a dipole flow [Milne-Thomson, 1960]. The flow field induced by recirculation wells differs somewhat from classic dipole flow because recirculation wells have finite radius, chamber length, and chamber separation and are placed in a specific setting necessitating consideration of the geometry of aquifer boundaries and hydrogeological conditions. In the following the term "dipole flow" will be used to denote the flow field induced in an aquifer (in the absence of ambient flow) by a combination of either a point source and sink or a uniformly distributed line source and sink, with finite separation between source and sink in both cases. Dipole flow provides an idealized model for the flow induced by recirculation wells [Zlotnik and Ledder, 1994].

Recirculation wells are currently being studied primarily in the context of two applications. First, recirculation wells are

Copyright 1996 by the American Geophysical Union.

Paper number 95WR03813.

0043-1397/96/95WR-03813\$02.00 used for aquifer remediation [Herrling et al., 1991; Herrling and Stamm, 1992; Gvirtzman and Gorelick, 1992, 1993; Falta, 1995]. In this area, characteristics of major interest are the geometry of the recirculation flow pattern [Herrling and Stamm, 1992; Philip and Walter, 1992; MacDonald and Kitanidis, 1993] and the head difference between the chambers as a function of pump flow rate, well parameters, and aquifer characteristics. Second, vertical circulation wells are the basis of a proposed method for determining aquifer parameters from analysis of the transient pressure head drawdown [Kabala, 1993; see also Kabala and Xiang, 1992].

The use of a recirculation well for aquifer characterization offers some advantages over the standard single-borehole techniques. In order to characterize spatial heterogeneity in hydraulic conductivity, hydraulic conductivity measurements on different spatial scales are needed. Typical examples of extensive studies at the Borden, Cape Cod, and Columbus sites of tracer tests combine pumping tests, slug tests, flowmeter tests, natural gradient tracer tests, use of permeameters, and grainsize analysis of disturbed samples [Sudicky, 1986; Hess et al., 1992; Rehfeldt et al., 1992]. With new developments in stochastic subsurface hydrology, the scale of measurements has become an important issue in theory and practice of contaminant hydrogeology. A need exists for the quantification of measurement scales for available aquifer testing methods and development of new techniques that would enable aquifer properties (horizontal and vertical hydraulic conductivities) to be determined at some given scale (see reviews by Molz et al. [1990], Dane and Molz [1991], Clausner [1992], and Kabala [1993]). All methods of analysis of data generated by the available tests are based on the solution of parabolic or elliptic partial differential equations for groundwater flow. Unlike the case of hyperbolic equations, which arise in fluid dynamics, the induced flow theoretically has an infinite extent of influence. Therefore only by designing special geometry for aquifer testing can one cre- 

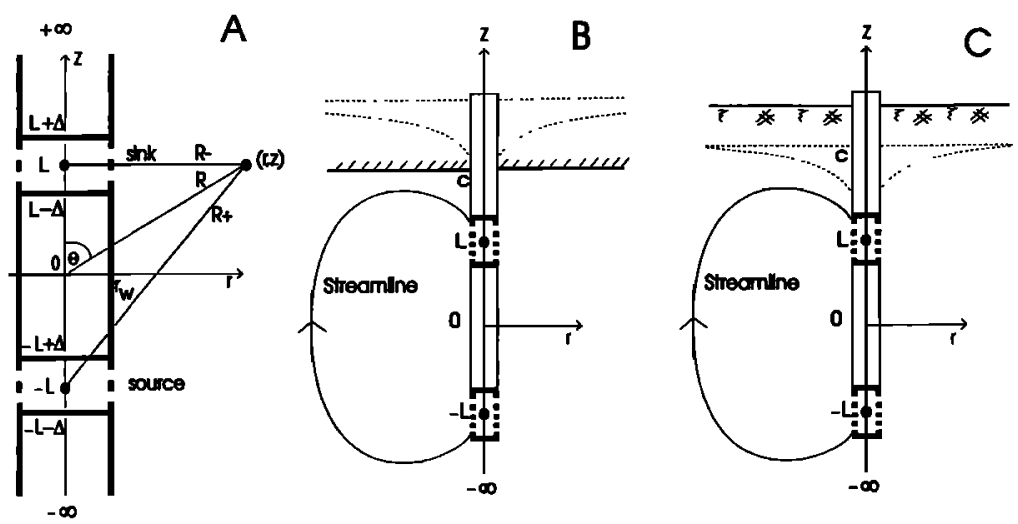

Figure 1. Schematic diagram of dipole in (a) infinite, (b) confined semi-infinite, and (c) unconfined semiinfinite aquifers.

ate flow patterns covering a limited region in the vicinity of the measuring device. Kabala [1993] has proposed the dipole flow test for measurement of horizontal hydraulic conductivity $K_{r}$, vertical hydraulic conductivity $K_{z}$, and specific storage coefficient $S_{s}$ at a predetermined scale.

Before the work of Kabala [1993], analytical solutions for the drawdown and flow field induced by a recirculation well were not available. Previously, results were obtained through computer-intensive numerical simulations including detailed finite element and boundary element modeling and particle tracking methods [Herrling et al., 1991; Herrling and Stamm, 1992; Philip and Walter, 1992; MacDonald and Kitanidis, 1993]. Kabala [1993] extended Hantush's [1961] analytical solution to determine the chamber drawdown in recirculation wells in a confined or leaky confined aquifer. However, an analytical description of the kinematic structure of dipole flow or analysis of drawdown near the borehole was not given. These problems are important both for theory and practice.

In this paper, new fully analytical expressions for drawdown and streamlines for dipole flow are derived. When aquifer boundaries are considered, the method of images [Muskat, 1937] will be used. Analytical description of the kinematic structure of the flow will be obtained using Stokes' stream function [Milne-Thomson, 1960; Bear, 1972]. In order to focus on the effect of the dipole, we will assume ambient flow is negligible and will start with the relatively simple case where there are no aquifer boundaries near the dipole. The effect of nearby horizontal boundaries will also be considered.

Applications of dipole flow theory will be given for typical recirculation wells where the chamber length is much larger than the well radius [Herling et al., 1991; Herling and Stamm, 1992; Kabala and Xiang, 1992; Kabala, 1993; MacDonald and Kitanidis, 1993]. One area of application is the analysis of spatial characteristics of zones where recirculation is intensive (the region of influence of the dipole). A second is the outline of a simple method for identification of hydraulic conductivities $K_{r}$ and $K_{z}$ from a few steady state dipole flow tests without transient measurements or computerized optimization.

\section{The Concept of the Region of Influence}

This section deals with the description of the region of influence of a dipole consisting of a point source and a point sink, which is similar to that considered by MacDonald and Kitanidis [1993].
A coordinate system is given in Figure 1a. The dipole well has radius $r_{w}$, chamber length $2 \Delta$, and chamber separation $2 D$. The distance between chamber centers is $2 L$, with $L=D+$ $\Delta$. The dipole-induced drawdown $s$ at any point in the aquifer is the difference between the contribution from the upper extraction chamber $s^{-}$(drawdown) and the lower injection chamber $s^{+}$(buildup):

$$
s=s^{-}-s^{+}
$$

For estimation of the drawdown in the far field of the flow and an introduction to the concept of the region of influence, we consider the dipole consisting of a point sink at $(0, L)$ and a point source at $(0,-L)$, with $\Delta=0$. In subsequent sections we extend the concept of region of influence to the more general case of a dipole with finite chamber length.

The drawdown at the point $(r, z)$ induced in an anisotropic, compressible, infinite (in both the radial and vertical directions) aquifer with specific storage coefficient $S_{s}$ by a continuous point sink (extraction) of strength $Q$ at $(0, L)$ is given by Carslaw and Jaeger [1959, pp. 257, 261]:

$$
\begin{aligned}
& s^{-}(r, z, t)=\frac{Q}{4 \pi K_{r} R_{-}} \operatorname{erfc}\left(\frac{\sqrt{S_{s}} R_{-}}{2 \sqrt{K_{z} t}}\right), \\
& R_{-}^{2}=\left(\frac{r}{a}\right)^{2}+(z-L)^{2}, \quad a^{2}=\frac{K_{r}}{K_{z}} .
\end{aligned}
$$

Similarly, the buildup induced by the source at $(0,-L)$ is

$$
\begin{aligned}
s^{+}(r, z, t) & =\frac{Q}{4 \pi K_{r} R_{+}} \operatorname{erfc}\left(\frac{\sqrt{S_{s}} R_{+}}{2 \sqrt{K_{z} t}}\right), \\
R_{+}^{2} & =\left(\frac{r}{a}\right)^{2}+(z+L)^{2} .
\end{aligned}
$$

In the case of an isotropic aquifer ( $a=1$ ), the values $R_{ \pm}$ represent the geometrical distances from the source and sink to the observation point $(r, z)$.

The drawdown at an arbitrary point for the transient case is given by formulas (1)-(3), and the maximum drawdown is achieved in the steady state:

$$
\begin{gathered}
s(r, z, t) \sim \frac{s_{r}}{2}\left(\frac{L}{R_{-}}-\frac{L}{R_{+}}\right), \quad \frac{4 K_{z} t}{S_{s} R^{2}} \gg 1, \\
R^{2}=\left(\frac{r}{a}\right)^{2}+z^{2}, \quad s_{r}=\frac{Q}{2 \pi K_{r} L} .
\end{gathered}
$$


After Taylor series expansion the drawdown for any point $(r, z)$ at a large distance from the dipole center is

$$
s(r, z, t) \sim s_{r} \frac{L^{2} z}{R^{3}}, \quad \frac{4 K_{z} t}{S_{s} R^{2}} \gg 1, \quad \frac{R}{L} \gg 1 .
$$

Thus the steady state drawdown along a ray ( $z / R$ fixed) induced by a dipole behaves as $R^{-2}$, instead of $R^{-1}$ as for the case of a single point source or sink. It can also be shown that for any point at a large distance from the dipole center, the vertical velocity component $V_{z}$ is approximately given by

$$
\begin{gathered}
V_{z}(r, z, t)=K_{z} \frac{\partial s}{\partial z} \sim \frac{L Q}{2 \pi a^{2} R^{3}}\left(1-3 \frac{z^{2}}{R^{2}}\right), \\
\frac{4 K_{z} t}{S_{s} R^{2}} \gg 1, \quad \frac{R}{L} \gg 1 .
\end{gathered}
$$

Thus the vertical velocity component vanishes with distance much faster than $R^{-2}$, which is the case for a single point source or sink.

Taking the half distance between chamber centers $L$ as a unit length scale, the radius of the region of influence can be estimated as a distance where the induced drawdown is roughly $1 \%$ of some reference value for the drawdown. The logical choice for a reference value is $s_{r}$ (given in (4)), which is the value obtained by the substitution $R=z=L$ in (5). Note that this is not actually the drawdown at the point $(0, L)$, since (5) is not valid for $R=L$. From (5),

$$
\frac{|s|}{s_{r}} \sim \frac{L^{2}|z|}{R^{3}}<\frac{L^{2}}{R^{2}}<\frac{a^{2} L^{2}}{r^{2}} .
$$

Thus the drawdown is certainly less than $1 \%$ of the reference value if

$$
r>10 a L \text { or }|z|>10 L
$$

Outside this zone, drawdown perturbations may be considered negligible. If the formula for the vertical velocity component (6) is selected for estimation of the influence radius, the region of influence will be even smaller. Note also that $s$ is never greater than when at steady state; thus the estimate (8) for the region of influence gives an upper bound for the region affected by the dipole. It is important to notice that anisotropy significantly affects only the horizontal extent of the region of influence. The concept of the region of influence and its size will be revisited by analysis of the flow structure utilizing stream functions.

An important question is what kind of boundary conditions should be used for determining drawdown invoked by dipole flow. A simple answer is suggested by the concept of the region of influence.

1. If the nearest aquifer boundary is far outside the region of influence, dipole flow can be described using a model of an aquifer of infinite extent.

2. If only one horizontal impermeable or low-permeable bed is near the region of influence, a model of a confined semi-infinite aquifer is appropriate.

3. If the water table is near the region of influence, but a horizontal confining lower bed is not, the unconfined semiinfinite aquifer model is appropriate. The drawdown induced by the dipole depends on the distance to the water table and should be calculated using an appropriate boundary condition at the moving surface.
4. If both upper and lower horizontal impermeable or lowpermeable boundaries are near the region of influence, $\mathrm{Kaba}$ la's [1993] aquifer model is appropriate.

5. If the water table and a lower horizontal confining bed are both near the region of influence, an unconfined aquifer model should be applied. Neuman's [1974] well functions can be utilized if the recirculation flow rate is small. Otherwise, more complex nonlinear flow models should be applied [MacDonald and Kitanidis, 1993].

In the sequel, we derive (with finite chamber length) well functions for an aquifer of infinite extent (case 1), a confined semi-infinite aquifer (case 2), and steady state flow in an unconfined semi-infinite aquifer (case 3 , simplified), and we develop of a more exact criterion for estimation of the region of influence of a dipole. Using Stokes' stream function, an analysis of flow structure induced by a dipole will be given.

A general numerical algorithm for determining aquifer parameters using analytical expressions for transient drawdown for case 4 (generalizable to cases 1 and 2) is given by Kabala [1993]. The analytical expressions derived herein are accurate and simple; the computational efficiency of Kabala's algorithm for transient drawdown is thereby increased for cases 1 and 2 .

For the sake of brevity, only expressions for the drawdown in the upper (extraction) chamber will be given; the formulas for drawdown in the lower (injection) chamber are analogous. For an infinite aquifer, drawdown induced by a dipole centered at $z=0$ is an odd function of $z$, so that formulas for the lower chamber may be obtained directly from formulas for the upper chamber.

\section{Dipole Flow in an Aquifer of Infinite Extent}

We now consider the drawdown induced by a dipole consisting of a linear source and sink uniformly distributed along the line $r=0$ over the lower and upper chambers, respectively. The drawdown is then governed by the equations

$$
\begin{gathered}
\frac{K_{r}}{r} \frac{\partial}{\partial r}\left(r \frac{\partial s}{\partial r}\right)+K_{z} \frac{\partial^{2} s}{\partial z^{2}}=S_{s} \frac{\partial s}{\partial t}, \\
\lim _{r \rightarrow 0} r \frac{\partial s}{\partial r}=\frac{Q}{4 \pi K_{r} \Delta} F(z), \\
F(z)=-1 \quad|z-L|<\Delta \\
F(z)=1 \quad|z+L|<\Delta \\
F(z)=0 \quad \text { otherwise }
\end{gathered}
$$

along with the condition $s=0$ as $r \rightarrow \infty, z \rightarrow \infty$, and $t=0$. The boundary condition (10) corresponds to a uniformly distributed line source and sink, rather than the point source and sink of section 2 .

\subsection{Local Transient Drawdown}

We iniroduce the scaied radial coordinate $\rho$ and parameter $q$, given by

$$
\rho=\frac{r}{a}, \quad q=\frac{Q}{4 \pi K_{r} \Delta},
$$

to simplify the notation for the problem (9) and (10). Solution for a linear sink only $(F(z)=-1,|z-L|<\Delta$, and $F(z)=$ 0 otherwise) was given by Hantush [1961, formula (2a)] for an aquifer of finite thickness. This solution can be significantly 
simplified for the aquifer of infinite thickness and transformed for the coordinate system given in Figure 1. Deriving a similar solution for a linear source $(F(z)=1,|z+L|<\Delta$, and $F(z)=0$ otherwise) and using the principle of superposition, one obtains the drawdown at any point $(r, z, t)$ induced by the dipole:

$$
\begin{aligned}
s= & \frac{q}{4}\left[M\left(\frac{\rho^{2}}{\tau}, \frac{z_{-+}}{\rho}\right)-M\left(\frac{\rho^{2}}{\tau}, \frac{z_{--}}{\rho}\right)\right. \\
& \left.+M\left(\frac{\rho^{2}}{\tau}, \frac{z_{+-}}{\rho}\right)-M\left(\frac{\rho^{2}}{\tau}, \frac{z_{++}}{\rho}\right)\right],
\end{aligned}
$$

where the function $M$ has been tabulated by Hantush [1961]:

$$
\begin{gathered}
M(u, x)=\int_{u}^{\infty} y^{-1} e^{-y} \operatorname{erf}(x \sqrt{y}) d y, \\
\tau=\frac{4 K_{z} t}{S_{s}}, \quad z_{ \pm \pm}=z \pm L \pm \Delta .
\end{gathered}
$$

\subsection{Upper Chamber Drawdown}

Hydraulic head measurements can be taken in the upper and lower chambers of a recirculation well. We define the upper chamber drawdown for a dipole $S(t)$ to be the vertical average of the drawdown at radius $r=r_{w}$, averaged over the screened portion of the upper chamber [Muskat, 1937]:

$$
S(t)=\frac{1}{2 \Delta} \int_{L-\Delta}^{L+\Delta} s\left(r_{w}, z, t\right) d z
$$

Substitution of (12) into (14), with use of the property $M(u,-x)=$ $-M(u, x)$ and the definition

$$
\begin{aligned}
& M_{1}(u, x) \equiv \int_{0}^{x} M(u, v) d v=x M(u, x)+\frac{2}{\sqrt{\pi u}}\left(e^{-u\left(1+x^{2}\right)}-e^{-u}\right) \\
& \quad+2\left(\operatorname{erfc} \sqrt{u}-\sqrt{1+x^{2}} \operatorname{erfc} \sqrt{u\left(1+x^{2}\right)}\right)
\end{aligned}
$$

results in a formula for $S(t)$ :

$$
\begin{aligned}
S(t) & =\frac{q \rho_{w}}{8 \Delta}\left[2 M_{1}\left(\frac{\rho_{w}^{2}}{\tau}, \frac{2 \Delta}{\rho_{w}}\right)-M_{1}\left(\frac{\rho_{w}^{2}}{\tau}, \frac{2 L-2 \Delta}{\rho_{w}}\right)\right. \\
& \left.+2 M_{1}\left(\frac{\rho_{w}^{2}}{\tau}, \frac{2 L}{\rho_{w}}\right)-M_{1}\left(\frac{\rho_{w}^{2}}{\tau}, \frac{2 L+2 \Delta}{\rho_{w}}\right)\right],
\end{aligned}
$$

where $\rho_{w} \equiv r_{w} / a$.

\subsection{Vertical and Horizontal Velocity Components}

Formulas for the vertical and horizontal velocity components are easily obtained from $V_{z}=K_{z} \partial s / \partial z$ and $V_{r}=$ $K_{r} \partial s / \partial r$ by differentiating (12) and using properties of the $M$ function [Hantush, 1964]:

$$
\begin{aligned}
V_{z}= & \frac{K_{z} q}{2}\left[\frac{1}{R_{-+}} \operatorname{erfc}\left(\frac{R_{-+}}{\tau^{1 / 2}}\right)-\frac{1}{R_{--}} \operatorname{erfc}\left(\frac{R_{--}}{\tau^{1 / 2}}\right)\right. \\
& \left.+\frac{1}{R_{+-}} \operatorname{erfc}\left(\frac{R_{+-}}{\tau^{1 / 2}}\right)-\frac{1}{R_{++}} \operatorname{erfc}\left(\frac{R_{++}}{\tau^{1 / 2}}\right)\right], \\
V_{r}= & \frac{K_{r} q}{2 r}\left[\frac{z_{--}}{R_{--}} \operatorname{erfc}\left(\frac{R_{--}}{\tau^{1 / 2}}\right)-\frac{z_{-+}}{R_{-+}} \operatorname{erfc}\left(\frac{R_{-+}}{\tau^{1 / 2}}\right)\right.
\end{aligned}
$$

$$
\begin{aligned}
& \left.+\frac{z_{++}}{R_{++}} \operatorname{erfc}\left(\frac{R_{++}}{\tau^{1 / 2}}\right)-\frac{z_{+-}}{R_{+-}} \operatorname{erfc}\left(\frac{R_{+-}}{\tau^{1 / 2}}\right)\right]+\frac{K, q}{2 r} e^{-r^{2} /\left(a^{2} \tau\right)} \\
& \cdot\left[\operatorname{erf}\left(\frac{z_{--}}{\tau^{1 / 2}}\right)-\operatorname{erf}\left(\frac{z_{-+}}{\tau^{1 / 2}}\right)+\operatorname{erf}\left(\frac{z_{++}}{\tau^{1 / 2}}\right)-\operatorname{erf}\left(\frac{z_{+-}}{\tau^{1 / 2}}\right)\right],
\end{aligned}
$$

with

$$
R_{ \pm \pm}^{2}=\rho^{2}+z_{ \pm \pm}^{2}=(r / a)^{2}+(z \pm L \pm \Delta)^{2} .
$$

\subsection{Steady State Results}

Of particular interest are formulas for the steady state drawdown, average drawdown, and velocity components. From (12) and (16)-(18), with the aid of the formula [Hantush, 1964, p. 318]

$$
M(0, x)=2 \sinh ^{-1} x=2 \ln \left(\sqrt{1+x^{2}}+x\right),
$$

one obtains the steady state local drawdown

$$
\begin{aligned}
\left.s_{\infty} \equiv s\right|_{t \rightarrow \infty}=\frac{q}{2}\left[\sinh ^{-1}\left(\frac{z_{-+}}{\rho}\right)-\sinh ^{-1}\left(\frac{z_{--}}{\rho}\right)\right. \\
\left.+\sinh ^{-1}\left(\frac{z_{+-}}{\rho}\right)-\sinh ^{-1}\left(\frac{z_{++}}{\rho}\right)\right] .
\end{aligned}
$$

(Note that for $\Delta / L \ll 1$, the steady state drawdown given by (21) reduces to the point source result (4).) Similarly, the steady state local velocities are

$$
\begin{aligned}
& V_{z, \infty}=\frac{K_{z} q}{2}\left[\frac{1}{R_{-+}}-\frac{1}{R_{--}}+\frac{1}{R_{+-}}-\frac{1}{R_{++}}\right], \\
& V_{r, \infty}=\frac{K_{r} q}{2 r}\left[\frac{z_{--}}{R_{--}}-\frac{z_{-+}}{R_{-+}}+\frac{z_{++}}{R_{++}}-\frac{z_{+-}}{R_{+-}}\right],
\end{aligned}
$$

and the steady state upper chamber drawdown is

$$
\begin{aligned}
S_{\infty}= & \frac{q}{2}\left[2 f\left(\frac{2 \Delta}{\rho_{w}}\right)+\frac{\rho_{w}}{\Delta}+\frac{2 L}{\Delta} f\left(\frac{2 L}{\rho_{w}}\right)\right. \\
& -\frac{L-\Delta}{\Delta} f\left(\frac{2 L-2 \Delta}{\rho_{w}}\right)-\frac{L+\Delta}{\Delta} f\left(\frac{2 L+2 \Delta}{\rho_{w}}\right),
\end{aligned}
$$

where the function

$$
f(x)=\sinh ^{-1} x-\frac{\sqrt{1+x^{2}}}{x},
$$

has the asymptotic property

$$
\begin{gathered}
f(x) \sim \operatorname{sgn}(x)(\ln 2|x|-1)+o\left(x^{-2}\right), \\
|x| \rightarrow \infty, \quad \operatorname{sgn}(x) \equiv \frac{x}{|x|} .
\end{gathered}
$$

The formula for the upper chamber drawdown can be simplified considerably when the well radius is much smaller than the half distance between chambers $D=L-\Delta$. Formula (26) may be used for all but the first occurrence of $f$ in (24); one obtains an asymptotic formula for $S_{\infty}$ valid for $\rho_{w} / D \ll 1$ :

$S_{\varkappa} \sim \frac{q}{2}\left[2 \ln \left(\frac{\sqrt{4 \Delta^{2}+\rho_{w}^{2}}+2 \Delta}{\rho_{w}}\right)-\frac{\sqrt{4 \Delta^{2}+\rho_{w}^{2}}}{\Delta}+\frac{\rho_{w}}{\Delta}\right.$ 


$$
\left.-\frac{L-\Delta}{\Delta} \ln \left(\frac{L-\Delta}{L}\right)-\frac{L+\Delta}{\Delta} \ln \left(\frac{L+\Delta}{L}\right)\right] .
$$

When the well radius is much smaller than both the half distance between chambers $D$ and the chamber half length $\Delta$, (26) may be used throughout (24); one obtains a simple expression for the upper chamber drawdown valid for $\rho_{w} / D \ll$ $1, \rho_{w} / \Delta \ll 1:$

$$
\begin{aligned}
S_{\infty} \sim q & {\left[\ln \left(\frac{4 \Delta}{\rho_{w}}\right)-1-\frac{L-\Delta}{2 \Delta} \ln \left(\frac{L-\Delta}{L}\right)\right.} \\
& \left.-\frac{L+\Delta}{2 \Delta} \ln \left(\frac{L+\Delta}{L}\right)\right] .
\end{aligned}
$$

\subsection{Stokes' Stream Function}

Stokes' stream function $\psi(r, z)$ is conveniently defined for steady state axisymmetric flow by the equations [MilneThomson, 1960; Bear, 1972]

$$
\begin{gathered}
\frac{\partial \psi}{\partial r} \equiv K_{z} r \frac{\partial s}{\partial z}=r V_{z, \infty}, \\
\frac{\partial \psi}{\partial z} \equiv-K_{r} r \frac{\partial s}{\partial r}=-r V_{r, \infty} \\
\psi(0,0)=0 .
\end{gathered}
$$

The utility of this function is directly apparent from its definition: the gradient of $\psi$ is orthogonal to the velocity in the $r z$-plane, so level curves of $\psi$ represent streamlines, and the upward discharge through a circle of arbitrary radius $r$ in the $z$ $=0$ plane is given directly by the value of Stokes' stream function at the point $(r, 0)$ :

$$
\begin{aligned}
& \int_{0}^{2 \pi} \int_{0}^{r} V_{z, \infty}(\bar{r}, 0) \bar{r} d \bar{r} d \theta=2 \pi \int_{0}^{r} \frac{\partial \psi}{\partial r}(\bar{r}, 0) d \bar{r} \\
& \quad=2 \pi \psi(r, 0) .
\end{aligned}
$$

To compute Stokes' stream function, we substitute (22) and (23) into (29) and (30) and obtain simple expressions for its derivatives:

$$
\begin{aligned}
\frac{\partial \psi}{\partial r} & =\frac{Q}{8 \pi a^{2} \Delta}\left[\frac{r}{R_{-+}}-\frac{r}{R_{--}}+\frac{r}{R_{+-}}-\frac{r}{R_{++}}\right] \\
& =\frac{Q}{8 \pi \Delta} \frac{\partial}{\partial r}\left[R_{-+}-R_{--}+R_{+-}-R_{++}\right], \\
\frac{\partial \psi}{\partial z} & =\frac{Q}{8 \pi \Delta}\left[\frac{z_{-+}}{R_{-+}}-\frac{z_{--}}{R_{--}}+\frac{z_{+-}}{R_{+-}}-\frac{z_{++}}{R_{++}}\right] \\
& =\frac{Q}{8 \pi \Delta} \frac{\partial}{\partial z}\left[R_{-+}-R_{--}+R_{+-}-R_{++}\right] .
\end{aligned}
$$

These formulas, along with (31), determine Stokes' stream function uniquely as

$$
\psi(r, z)=\frac{Q}{8 \pi \Delta}\left[4 \Delta+R_{-+}-R_{--}+R_{+-}-R_{++}\right] .
$$

For convenience, we define the normalized Stokes' stream function
$\Psi(r, z)=\frac{\psi(r, z)}{\psi(\infty, 0)}=1-\frac{R_{--}-R_{-+}+R_{++}-R_{+-}}{4 \Delta}$

Then the surface that confines the fraction $\gamma$ of the flow is given by $\Psi(r, z)=\gamma$ or

$R_{--}-R_{-+}+R_{++}-R_{+-}=4 \Delta(1-\gamma), \quad 0 \leq \gamma \leq 1$.

Although Stokes' stream function has been defined in terms of the physical spatial coordinates $(r, z)$, illustrations of the streamlines given by (37) can also be plotted with the normalized spatial coordinates $(\rho, z)$.

Formula (37) allows for the characterization of zones around the borehole according to the intensity of flow circulation. For $\gamma \rightarrow 0$ the surface defined by (37) confines a vanishingly small volume of aquifer. For $\gamma \rightarrow 1$ this surface expands outward and can be used to define the region of influence of the dipole. The simple analytical expression (37) allows determination of the shape of the surface bounding any percentage of the flow, without need for intensive numerical computations, as in previous studies [Herrling et al., 1991; Herrling and Stamm, 1992; Philip and Walter, 1993].

\section{Influence of Aquifer Boundaries}

We now consider briefly the case in which the dipole is located near an aquifer boundary; specifically, we assume a boundary at $z=c$ (Figures $1 \mathrm{~b}$ and $1 \mathrm{c}$ ). In this section a subscript $I$ will be used to denote any results from the infinite aquifer model of the previous section.

If the boundary at $z=c$ represents a confining layer, then there is no flow across the boundary. The transient drawdown $s_{C}$ at an arbitrary point of the semi-infinite aquifer is the solution of the problem given by (9) and (10) and the additional conditions

$$
\begin{gathered}
s_{C}(\rho,-\infty, t)=s_{C}(\infty, z, t)=s_{C}(\rho, z, 0)=0, \\
\frac{\partial s_{C}}{\partial z}(\rho, c, t)=0 .
\end{gathered}
$$

The solution to this problem for drawdown can be obtained from the drawdown in an infinite aquifer (12) by adding a contribution from an antisymmetric dipole (mirror image with respect to the $z=0$ plane). The center of the image dipole is located at $z=2 c$; thus

$$
s_{C}(\rho, z, t)=s_{I}(\rho, z, t)-s_{I}(\rho, z-2 c, t) .
$$

The normalized Stokes' stream function is obtained in the form

$$
\Psi_{C}(r, z)=\Psi_{I}(r, z)-\Psi_{I}(r, z-2 c),
$$

where $\Psi$, is given by (36). Formulas for velocity components and the steady state formulas for drawdown and velocity components can also be obtained by superposition. According to formula (38), the impermeable boundary will cause the drawdown at $z=c$ to be doubled compared to the same point in an infinite aquifer (since $s_{I}(\rho,-z, t)=-s_{I}(\rho, z, t)$ ), while at other points the mirror dipole will add a positive correction term smaller in magnitude than the infinite aquifer term $s_{I}(\rho, z, t)$.

The formula for the transient upper chamber drawdown can be obtained from (14) and (39): 


$$
\begin{aligned}
S_{C}(t)= & S_{I}(t)+\frac{q \rho_{w}}{8 \Delta}\left[M_{1}\left(\frac{\rho_{w}^{2}}{\tau}, \frac{2 c-2 L-2 \Delta}{\rho_{w}}\right)\right. \\
& +M_{1}\left(\frac{\rho_{w}^{2}}{\tau}, \frac{2 c-2 L+2 \Delta}{\rho_{w}}\right)-2 M_{1}\left(\frac{\rho_{w}^{2}}{\tau}, \frac{2 c-2 L}{\rho_{w}}\right) \\
& -M_{1}\left(\frac{\rho_{w}^{2}}{\tau}, \frac{2 c-2 \Delta}{\rho_{w}}\right)+2 M_{1}\left(\frac{\rho_{w}^{2}}{\tau}, \frac{2 c}{\rho_{w}}\right) \\
& \left.-M_{1}\left(\frac{\rho_{w}^{2}}{\tau}, \frac{2 c+2 \Delta}{\rho_{w}}\right)\right]
\end{aligned}
$$

where $S_{I}(t)$ is given by formula (16).

The steady state upper chamber drawdown $S_{C_{\infty}}$ is obtained from (41) with $\tau \rightarrow \infty$. In practice, $\rho_{w} /(c-L-\Delta) \ll 1$, so the asymptotic result (26) may be applied. The final formula for upper chamber drawdown is then

$$
\begin{aligned}
S_{C \infty} \sim & S_{J \infty}+\frac{q}{2}\left[\frac{c-L-\Delta}{\Delta} \ln \left(1-\frac{\Delta}{c-L}\right)\right. \\
& +\frac{c-L+\Delta}{\Delta} \ln \left(1+\frac{\Delta}{c-L}\right)-\frac{c-\Delta}{\Delta} \ln \left(1-\frac{\Delta}{c}\right) \\
& \left.-\frac{c+\Delta}{\Delta} \ln \left(1+\frac{\Delta}{c}\right)\right]
\end{aligned}
$$

where $S_{1 \infty}$ is given by (27) or (28).

The cases of an unconfined semi-infinite aquifer and an aquifer of finite thickness should be treated separately [e.g., Dagan, 1967; Neuman, 1974; MacDonald and Kitanidis, 1993; Zlotnik and Ledder, 1994].

\section{Results and Discussion}

The analytical formulas for dipole flow characteristics are remarkably simple and important for both recirculation well design and aquifer parameter estimation. Of particular significance are formula (28) for steady state upper chamber drawdown and formula (37) for streamlines.

Formula (28) for upper chamber drawdown is simpler than Kabala's [1993] infinite series formula, which has modest convergence. It lends itself to analysis of such factors affecting upper chamber drawdown as hydraulic conductivity, anisotropy, and dipole design parameters.

Results for streamlines previously could be obtained only by the use of numerical methods. Herrling and Stamm [1992] used 200,000 finite elements, Philip and Walter [1992] used a particle tracking algorithm, and MacDonald and Kitanidis [1993] used the boundary element technique. The new formulas expressed through elementary functions provide both insight into the role of aquifer parameters in dipole flow and an important computational tool to approximate results of previous numerical simulations

\subsection{Flow Field in an Infinite Aquifer}

The geometry of the region of intensive recirculation is of particular interest. Figure 2 illustrates the steady state flow induced by a dipole in an infinite aquifer. The solid curves are the streamlines marking out $70 \%, 80 \%$, and $90 \%$ of the flow computed from formula (37). The dashed curves are equipotentials, computed from formula (21). Two different values of $\Delta / L$ were used ( 0.2 for Figure $2 \mathrm{a}$ and 0.8 for Figure $2 \mathrm{~b}$ ) in order to show the insensitivity of the far-field flow to the screen

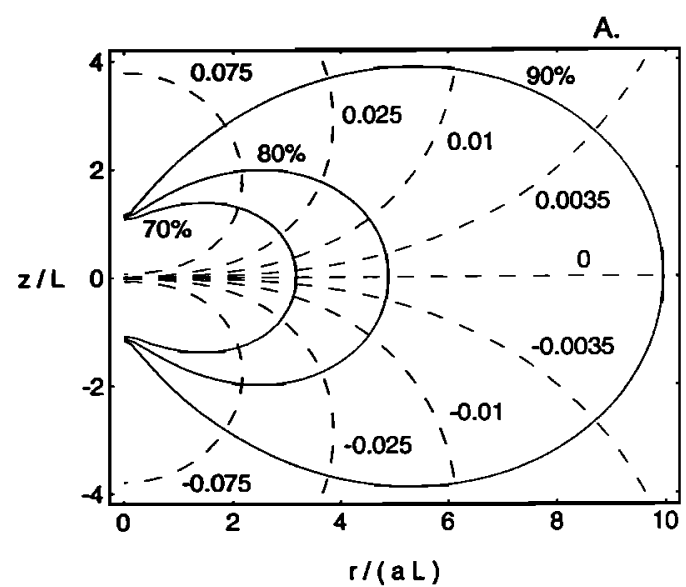

B.

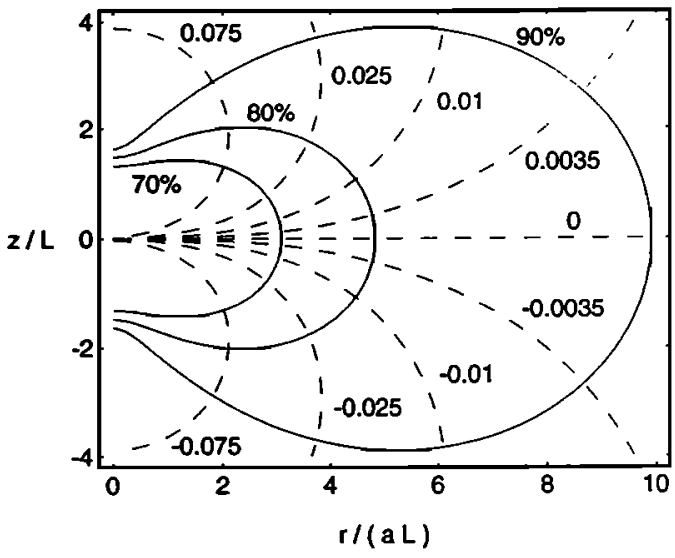

Figure 2. Flow around dipole with different chamber length $\Delta$ in infinite aquifer. Solid curves indicate streamlines confining the given percentage of the flow. Dashed lines indicate equipotentials. (a) $\Delta=0.2 L$, (b) $\Delta=0.8 L$.

half-length $\Delta$. Even the $70 \%$ streamline is essentially unaffected by the choice of $\Delta$. The influence region of the dipole, in physical coordinates, has approximately the shape of a torus, with a cross section that is roughly elliptical far from the $z$ axis and has horizontal major axis of length $10 a \mathrm{~L}$ and vertical minor axis of length $8 L$. In the immediate vicinity of the dipole the streamlines are hyperbolas with foci at the ends of the extraction and injection chambers (see work by MilneThomson [1960, p. 458]). In the absence of aquifer boundary effects, points having $|z|>4 L$ are outside the region of noticeable dipole flow. The small region of influence supports Kabala's [1993] suggestion to use a dipole to measure local values of aquifer parameters.

Figure 3 illustrates the effect of changes in chamber halflength $\Delta$ on the near field of the dipole flow. The curves depict the steady state upper chamber drawdown as a function of the ratio of chamber length to the distance between chamber centers for various values of $\rho_{w} / L$, using formulas (24) and (28) (one should notice the use of $s_{r}$, rather than $q$, for the drawdown scale in the figure). Unlike in the case of stream function and streamline computations, upper chamber drawdown is significantly affected by dipole design parameters. The approximation of a dipole as a combination of point source and point sink [MacDonald and Kitanidis, 1993] should be used with 
caution. Note that for the ranges of parameter values used in Figure 3, the simple asymptotic formula (28) provides a very good approximation to the more complicated formula (24).

\subsection{Flow Field in a Semi-Infinite Aquifer}

The steady state flow field induced by a dipole in a semiinfinite aquifer is compared to that for an infinite aquifer in Figure $4 \mathrm{a}$. The $70 \%, 80 \%$, and $90 \%$ streamlines for aquifers with no-flow boundaries at $c=2 L, 5 L, \infty$ are compared using formula (40). We see that a no-flow boundary near the dipole has the effect of depressing and compressing the region of influence. Worthy of note is the observation that a no-flow boundary located just outside the region of influence of a dipole (the $c=5 L$ example) affects only the flow significantly outside the $80 \%$ streamline. When the confining layer is inside the region of influence of the dipole, as is the case with $c=$ $2 L$, then the flow pattern is significantly changed.

Figure $4 \mathrm{~b}$ further illustrates the steady flow pattern for a dipole with a no-flow boundary at $c=5 L$, just outside the region of influence (formulas (39) and (40)). Comparison with the flow field for a dipole in an infinite aquifer (Figure 2) shows that the flow in the lower portion of the aquifer $(z<0)$ is not as strongly affected by the boundary as the flow in the upper portion. This is clearly to be expected from the conceptualization of the flow as equivalent to that produced by superposition of the physical dipole with a mirror dipole located above the confining layer. The effect of the mirror dipole decays with depth like $1 / R^{2}$, as in (5).

The transient effect of a confining layer on dipole flow is illustrated in Figure 5 . In both Figures $5 \mathrm{a}$ and $5 \mathrm{~b}$ the time is given with respect to the timescale

$$
t_{r}=S_{s} L^{2} / K_{z} \text {. }
$$

The drawdown in the far field is examined in Figure 5a with a comparison of the results for infinite (formula (12)) and confined (formula (39)) aquifers for points roughly located near the apex of the $70 \%, 80 \%$, and $90 \%$ streamlines. The confining layer does not affect the flow in the earliest stages; the degree to which the flow is ultimately affected depends on the distance from the point being considered to the center of the mirror dipole at $(0,2 c)$.

The transient effect of the confining layer on the upper chamber drawdown is shown in Figure 5b. Here we see that the effect of a confining layer located at $c=5 L$ is immeasurably small, and even the effect of a layer at $c=2 L$ is not significant.

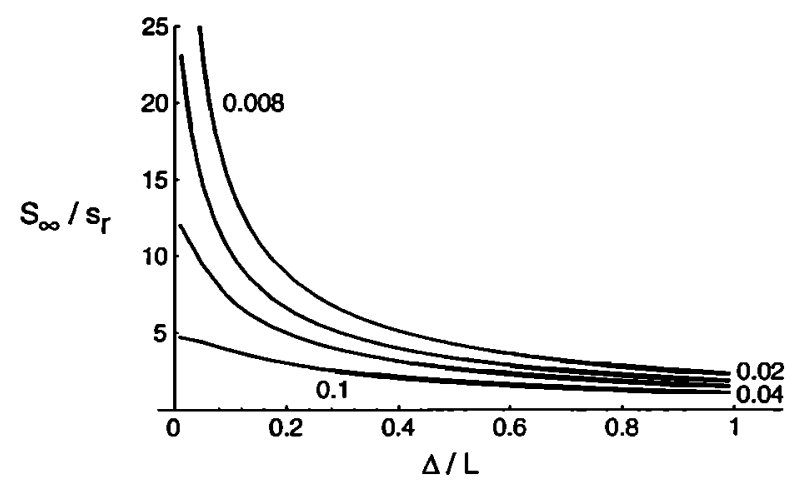

Figure 3. Dimensionless steady drawdown $S_{\infty} / s_{r}$ in single chamber as a function of chamber half length $\Delta / L$ for different values of dimensionless chamber radius $r_{w} /(a L)$.
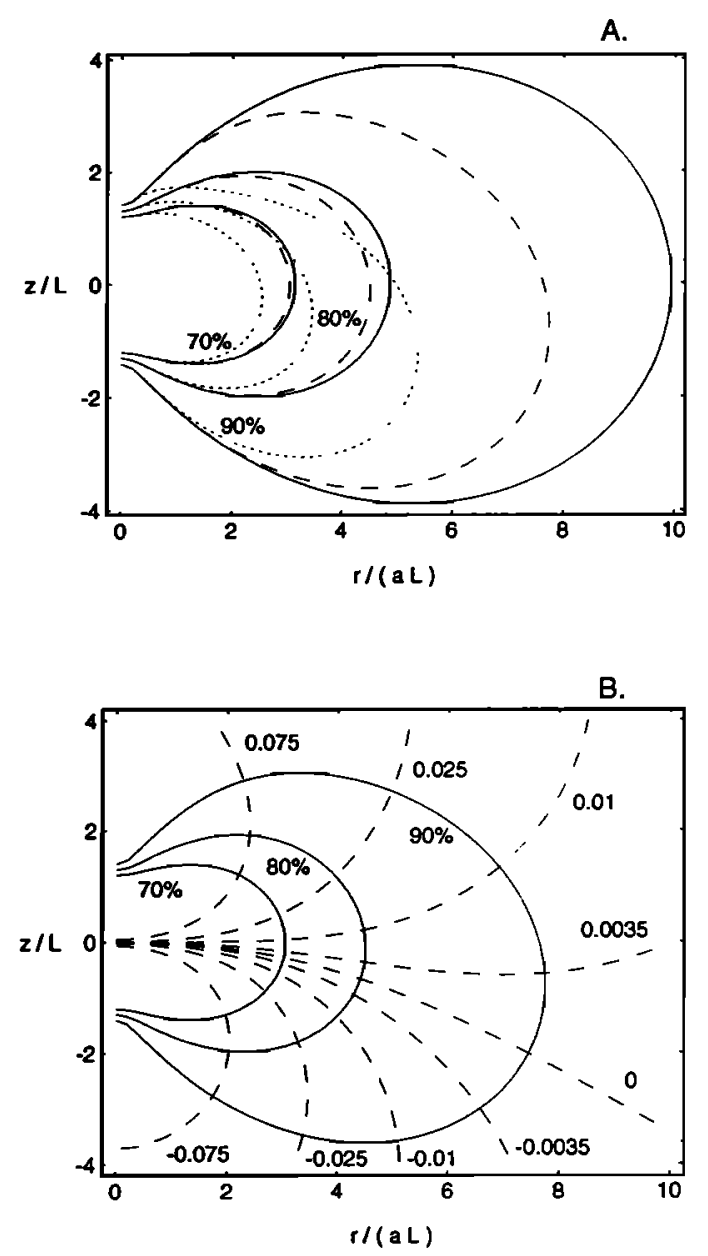

Figure 4. Effect of impermeable boundary location on steady groundwater flow around dipole $(\Delta=0.5 L)$. (a) Position of $70 \%, 80 \%$, and $90 \%$ flow streamlines for the distance of dipole center from the boundary $c=2 L$ (dotted lines), $c=5 L$ (dashed lines), and $c=\infty$ (solid lines). (b) streamlines (solid lines) and equipotentials (dashed lines) for $c=5 L$.

Note also that the steady state is reached rather quickly. For sandy aquifers $\left(S_{s}=0.0001-0.0011 / \mathrm{m}, K_{z}=0.00001-0.0001\right.$ $\mathrm{m} / \mathrm{s}$ ) with $L=1-5 \mathrm{~m}$, the reference time is $t_{r}=1-2500 \mathrm{~s}$. Therefore the right edge of Figure $5 \mathrm{~b}$ can be generally reached within a few hours, and often much faster.

\subsection{Steady State Dipole Flow Test}

Technically, a dipole flow test can be performed in wells with screen diameter as small as $r_{w}=0.05 \mathrm{~m}$. In most aquifers the anisotropy coefficient $a$ is at least 1 , so for chamber separation $2 D=1 \mathrm{~m}$, and screen length on the order of $1 \mathrm{~m}$, we have $\rho_{w} / \Delta$ and $\rho_{w} / D$ on the order of 0.1 . From (26) the error caused by applying (28) is on the order of the squares of these ratios $(1 \%)$; hence $(28)$ is sufficiently accurate for practical use. The formulas can be rewritten as

$$
\begin{gathered}
S_{\infty} \sim \frac{Q}{4 \pi K_{r} \Delta} \ln \left[\frac{4 a \Phi(\lambda) \Delta}{e r_{w}}\right], \\
\Phi(\lambda)=\left(\frac{\lambda^{2}}{\lambda^{2}-1}\right)^{\lambda / 2}\left(\frac{\lambda-1}{\lambda+1}\right)^{1 / 2}, \quad \lambda=\frac{L}{\Delta}=1+\frac{D}{\Delta},
\end{gathered}
$$

where $e=2.7182 \cdots$. The function $\Phi$ (shown in Figure 6) increases monotonically from 0.5 to 1.0 as $\lambda$ increases from 1 to $\infty$. 

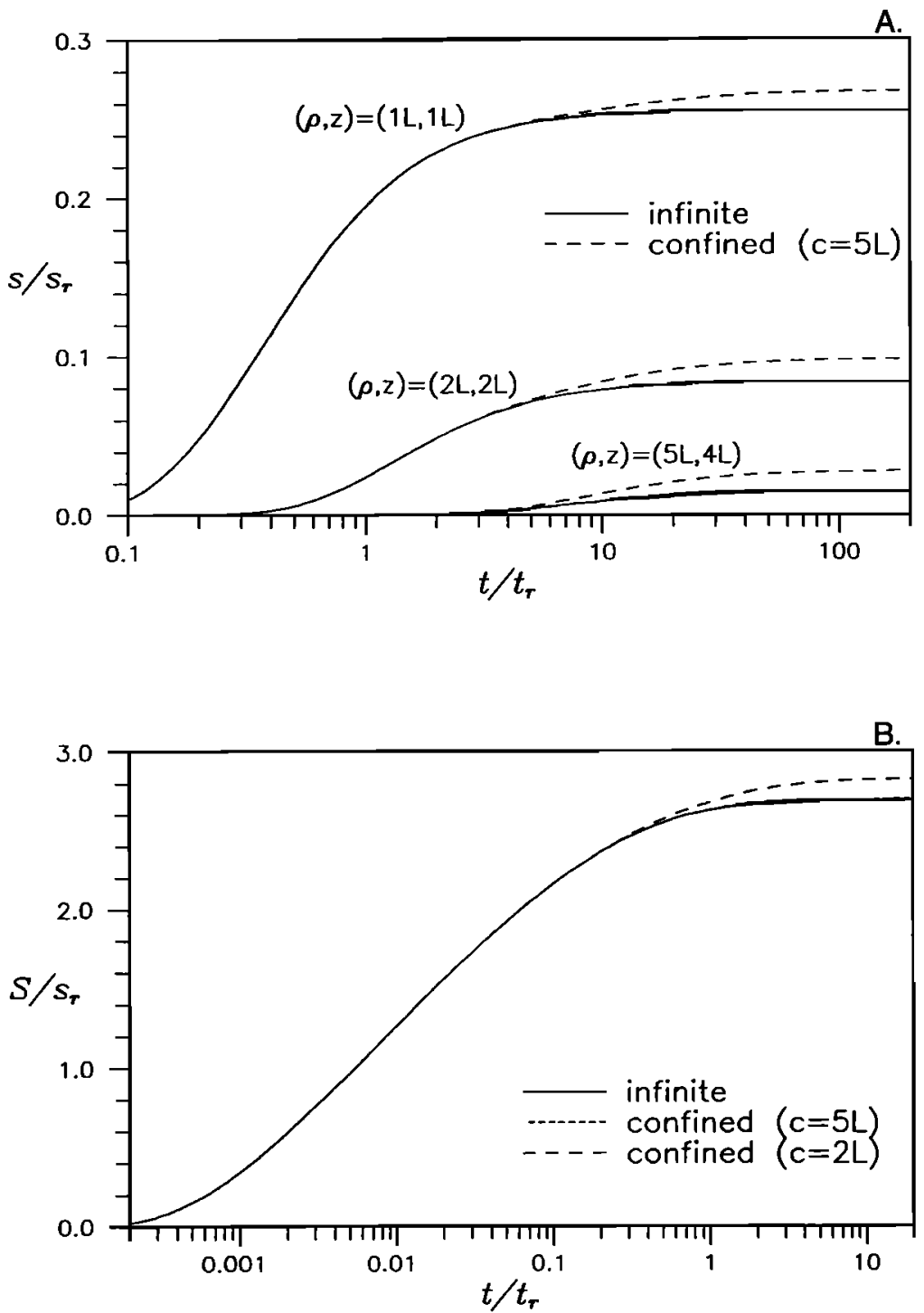

Figure 5. Transient drawdown induced by a dipole $(\Delta=0.5 L)$ in an infinite aquifer (solid line) and a confined semi-infinite aquifer (dashed line) with boundary at the distance $c=5 L$ from dipole center. (a) Local drawdown $s(r, z, t) / s_{r}$ at the points $(\rho, z)=(L, L),(2 L, 2 L)$, and $(5 L, 4 L)$ as a function of dimensionless time. (b) Drawdown $S(t)$ in a single chamber with dimensionless radius $r_{w} /(a L)=0.04$ corrected for anisotropy in infinite and semi-infinite aquifers.

This formula clearly shows logarithmic dependence of upper chamber drawdown on the anisotropy ratio $a$. This readily explains results obtained numerically [Herrling et al., 1991, Figure 9b; Kabala, 1993, Figure 3].

As an example of possible application to a dipole flow test, consider a deep alluvial sand and gravel aquifer with horizontal hydraulic conductivity $K_{r}=100 \mathrm{~m} /$ day and anisotropy ratio given by $a^{2}=K_{r} / K_{z}=9$. A flow rate of $Q=50 \mathrm{~m}^{3} /$ day is common for small submersible pumps. Consider a pair of tests in a well of radius $r_{w}=0.1 \mathrm{~m}$ employing a constant central packer length $(D=1.0 \mathrm{~m})$ and two chamber lengths $\left(\Delta_{1}=0.3\right.$ $\mathrm{m}, \Delta_{2}=0.5 \mathrm{~m}$ ). The corresponding values of upper chamber drawdown will be $S_{1}=0.33 \mathrm{~m}, S_{2}=0.23 \mathrm{~m}$. For an isotropic aquifer $(a=1)$ the corresponding drawdown values will be $S_{1}=0.18 \mathrm{~m}, S_{2}=0.15 \mathrm{~m}$. Even for highly conductive materials, these drawdown values are easily detectable and sensitive enough to changes in the values of the control parameter.

The steady state dipole flow test allows for flexible design. Two dipole design parameters $(\Delta, \lambda)$ control the steady state upper chamber drawdown. It is therefore possible to fix the chamber length $2 \Delta$, the central packer length $2 D$, or the ratio $D / \Delta$ while varying the other two of these quantities, or it is possible to vary all three of these quantities in order to maximize the range of the product $\Phi(\lambda) \Delta$ that appears inside the natural logarithm of (44).

For example, using chamber length as a control parameter, one could perform two dipole flow tests. For chamber lengths $2 \Delta_{1}$ and $2 \Delta_{2}$ and fixed length of central packer, the corresponding steady state upper chamber drawdowns $S_{1}$ and $S_{2}$ are measured. From direct substitution into (44), the parameters of interest are given by the formulas

$$
\begin{gathered}
K_{r}=\frac{Q}{4 \pi\left(S_{2} \Delta_{2}-S_{1} \Delta_{1}\right)} \ln \left[\frac{\Phi\left(\lambda_{2}\right) \Delta_{2}}{\Phi\left(\lambda_{1}\right) \Delta_{1}}\right], \\
a=\frac{r_{w}}{4 \Phi\left(\lambda_{t}\right) \Delta_{l}} \exp \left[1+\frac{4 \pi K_{r} S_{t} \Delta_{t}}{Q}\right], \quad i=1,2 .
\end{gathered}
$$

The estimate of anisotropy $a$ is more sensitive to measurement 


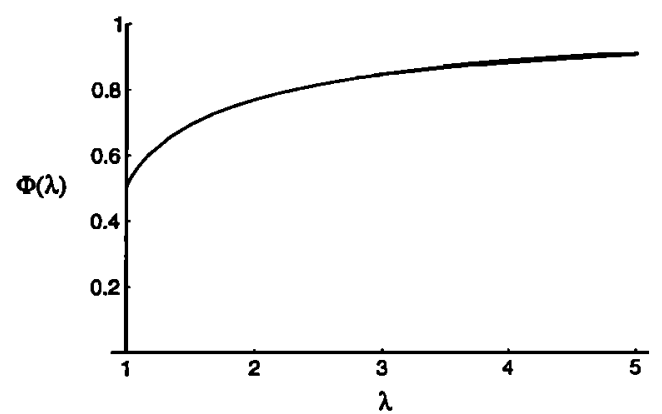

Figure 6. The dipole shape function $\Phi(\lambda)$.

errors than the estimate of $K_{r}$, similar to the results of Kabala [1993, p. 105]. Using the design parameters $Q, r_{w}, D, \Delta_{1}$, and $\Delta_{2}$ from the previous example, with experimental measurements $S_{1}=0.34 \mathrm{~m}, S_{2}=0.24 \mathrm{~m}$ (allowing for a small measurement error), one obtains the results $K_{r}=101 \mathrm{~m} /$ day, $a=3.3$. With more than two measurements, one might use the prediction (from (44)) that a graph of $S_{\infty} \Delta$ versus in $[\Phi(\lambda) \Delta]$ should be linear.

Owing to the diversity of hydrogeological conditions, the uniform anisotropic aquifer model may not be applicable in some situations of practical interest. Dipole test results can be strongly affected by natural aquifer heterogeneity and the skin effect. In particular, dipole flow tests can be more sensitive to skin than other single-borehole methods that invoke mainly horizontal flow.

\section{Conclusions}

Dipole flow is the flow induced in an aquifer with negligible ambient flow by a combination of a uniformly distributed line (or point) source and a sink having the same geometry, located on the $z$ axis and separated by a finite length $2 L$. The region of influence of a dipole may be taken to be the region within the stream surface containing $90 \%$ of the flow. With this definition the region of influence of a dipole extends to approximately $10 a L$ in the radial direction and $4 L$ in the vertical direction (from the dipole center), where $a$ is the anisotropy ratio $\left(K_{r} / K_{z}\right)^{1 / 2}$. The spatial scale over which intensive water circulation occurs in the aquifer can be controlled by modification of the distance between the chamber centers and to a lesser extent by the chamber length.

When considering the drawdown and flow field produced by a dipole in an aquifer, several different models may be employed, differing in the treatment of aquifer boundaries. If the dipole is placed so that no boundary is near the region of influence as described above, then the infinite aquifer model can be conveniently used. Both transient and steady state formulas for the drawdown are given in terms of known functions that can be efficiently computed. Use of the infinite aquifer model offers a significant reduction in computational efforts for approximating the flow in the vicinity of a recirculation well.

The infinite aquifer model is easily generalized to the case of a single horizontal impermeable boundary located within the region of influence of the dipole. This semi-infinite model also applies for the steady state solution in the case where the water table (but no lower horizontal boundary) is located within the region of influence. Both the radial and vertical extent of the region of influence are reduced by the presence of a horizontal boundary near the region of influence [Zlotnik and Ledder, 1994].

When two horizontal impermeable boundaries are located within the region of influence of a dipole, Kabala's [1993] confined aquifer model with Hantush's [1961] well function can be applied. If the water table and a lower horizontal impermeable boundary are located within the region of influence and the water table inflection is small, then an unconfined aquifer model should be used with the well function of $\mathrm{Neu}$ man [1974]. Otherwise, more complex nonlinear models should be applied [MacDonald and Kitanidis, 1993].

Analytical expressions for Stokes' stream functions were derived and applied for dipole flow analysis in subsurface flow. These functions efficiently describe the streamlines in the flow and can be used to delineate regions with intensive water circulation in aquifers with negligible ambient flow. Examples of the flow field determined with Stokes' stream function show that the dipole chamber length can be neglected in estimation of the region of influence, but it is important in estimation of the flow in the immediate vicinity of the dipole chambers and particularly for the chamber drawdown.

For the practically important case when the chamber length is much larger than the well radius, simple formulas show the dependence of the upper chamber drawdown on the chamber length, radius, and interchamber distance. The steady state drawdown is seen to be logarithmicly dependent on the anisotropy ratio. Methods for identifying the horizontal hydraulic conductivity and the anisotropy ratio might be based on the formulas for steady state upper chamber drawdown.

\section{Notation}

$a$ anisotropy ratio, where $a^{2}=K_{r} / K_{z}$ (dimensionless).

c location of horizontal aquifer boundary relative to the dipole center $(\mathrm{L})$.

$D$ half distance between chambers, where $D=$ $L-\Delta(\mathrm{L})$.

$F(z)$ function for description of sinks and sources (dimensionless).

$K_{r}, K_{z}$ horizontal and vertical hydraulic conductivities (L/T).

$L$ half distance between dipole chamber centers (L).

$M, M_{1}$ Hantush well-function and its definite integral (dimensionless).

$Q$ discharge of extraction chamber of dipole (linear or point) $\left(\mathrm{L}^{3} / \mathrm{T}\right)$.

$q$ discharge parameter, where $q=Q / 4 \pi K_{r} \Delta(\mathrm{L})$.

$r$ radial coordinate $(\mathrm{L})$.

$r_{w}$ well radius (L).

$R_{ \pm}, R_{ \pm \pm}$distances from different characteristic points of the dipole, corrected for anisotropy (L).

$s$ local drawdown in the aquifer (L).

$s_{r}$ reference drawdown, where $s_{r}=Q / 2 \pi K_{r} L(\mathrm{~L})$.

$s^{ \pm}$drawdown (-) in extraction chamber and buildup ( \pm ) in injection chamber (L).

$S$ vertical average of drawdown over the upper chamber $(\mathrm{L})$.

$S_{s}$ specific storage coefficient $(1 / \mathrm{L})$.

$t$ time.

$V_{r}, V_{z}$ Darcy velocity components in cylindrical coordinates $(\mathrm{L} / \mathrm{T})$. 
$z$ vertical coordinate measured upward from dipole center $(\mathrm{L})$.

$z_{ \pm \pm}$vertical distances from different characteristic points of dipole (L).

$\gamma$ fraction of total flow within a given streamline (dimensionless).

$\Delta$ half length of dipole chamber (L).

$\lambda$ ratio of interchamber distance to chamber length, where $\lambda=L / \Delta$ (dimensionless).

$\rho$ radial coordinate corrected for anisotropy, where $\rho=r / a(\mathrm{~L})$.

$\rho_{w}$ well radius corrected for anisotropy, where $\rho_{w}=r_{w} / a(\mathrm{~L})$.

$\tau$ parameter, where $\tau=4 K_{z} t / S_{s}\left(\mathrm{~L}^{2}\right)$.

$\Phi(\lambda)$ dipole shape function (dimensionless).

$\psi$ Stokes' stream function $\left(\mathrm{L}^{3} / \mathrm{T}\right)$.

$\Psi$ normalized Stokes' stream function (dimensionless).

Acknowledgments. The authors wish to thank Z. Kabala for discussions about the dipole flow test. This work was supported by the Water Center of the University of Nebraska-Lincoln, an annual federal matching grant as authorized by the section 104 of the Water Resources Research Act, the National Water Research Institute, and the Research Council of the University of Nebraska-Lincoln. Development of software for the RISC-6000 system was partially supported by IBM.

\section{References}

Bear, J., Dynamics of Fluids in Porous Media, 764 pp., Elsevier, New York, 1972.

Carslaw, H. S., and J. C. Jaeger, Conduction of Heat in Solids, 510 pp., Clarendon, Oxford, 1959.

Clausner, C., Permeability of crystalline rocks, Eos Trans. AGU, 73(21), 232, 1992.

Dagan, G., A method of determining the permeability and effective porosity of unconfined aquifers, Water Resour. Res., 3(4), 1059-1071, 1967.

Dane, J. H., and F. J. Molz, Physical measurements in subsurface hydrology, U.S. Natl. Rep. Int. Union Geod. Geophys., 1987-1990, Rev. Geophys., 29, 270-279, 1991.

Falta, R. F., Analytical solutions for gas flow due to gas injection and extraction from horizontal wells, Ground Water, 33(2), 235-246, 1995.

Gvirtzman, H., and S. M. Gorelick, The concept of in situ vapor stripping for removing VOCs from groundwater, Transp. Porous Media, 8(1), 71-92, 1992.

Gvirtzman, H., and S. M. Gorelick, Using air-lift pumping as an in situ aquifer remediation technique, Water Sci. Technol., 27(7-8), 195201, 1993.

Hantush, M. S., Drawdown around a partially penetrating well, $J$. Hydraul. Div. Am. Soc. Civ. Eng., 87(HY4), 83-98, 1961.

Hantush, M. S., Hydraulics of wells, in Advances in Hydroscience, vol. 1, edited by V. T. Chow, pp. 281-432, Academic, San Diego, Calif., 1964.
Herrling, B., and J. Stamm, Numerical results of calculated 3D vertical circulation flows around wells with two screen sections for in situ on-site aquifer remediation, in Computational Methods in Water Resources $L X$, vol. I, Numerical Methods in Water Resources, edited by T. F. Russel, R. E. Ewing, C. A. Brebbia, W. G. Gray, and G. F. Pinder, pp. 483-492, Elsevier Sci., New York, 1992.

Herrling, B., J. Stamm, and W. Buerman, Hydraulic circulation system for in-situ bioreclamation and/or in situ remediation of strippable contamination, in In Situ Bioreclamation: Applications and Investigations for Hydrocarbon and Contaminated Site Remediation, edited by R. E. Hinchie and R. F. Olfenbuttel, pp. 173-195, ButterworthHeinemann, Newton, Mass., 1991.

Hess, K. M., S. H. Wolf, and M. A. Celia, Large-scale natural gradient tracer test in sand and gravel, Cape Code, Massachusetts, 3, Hydraulic conductivity variability and calculation macrodispersivities, Water Resour. Res., 28(8), 2011-2027, 1992.

Kabala, Z. J., Dipole flow test: A new single-borehole test for aquifer characterization, Water Resour. Res., 29(1), 99-107, 1993.

Kabala, Z. J., and J. Xiang, Skin effect and its elimination for single borehole aquifer tests, in Computational Methods in Water Resources $L X$, vol. I, Numerical Methods in Water Resources, edited by T. F. Russel, R. E. Ewing, C. A. Brebbia, W. G. Gray, and G. F. Pinder, pp. 467-474, Elsevier Sci., New York, 1992.

MacDonald, T. P., and P. K. Kitanidis, Modeling of the free surface of an unconfined aquifer near recirculation well, Ground Water, 31(5), 774-780, 1993.

Milne-Thomson, L. M., Theoretical Hydrodynamics, 660 pp., McMillan, New York, 1960

Molz, F. J., O. Guven, J. G. Melville, and C. Cardone, Hydraulic conductivity measurement at different scales and contaminant transport modeling, in Dynamics of Fluids in Hierarchial Porous Media, chap. 3, edited by J. Cushman, pp. 37-59, Academic, San Diego, Calif., 1990.

Muskat, M., The Flow of Homogeneous Fluids Through Porous Medıa, McGraw-Hill, New York, 1937.

Neuman, S. P., Effect of partial penetration on flow in unconfined aquifers considering delayed gravity response, Water Resour. Res., 10(2), 303-312, 1974.

Philip, R. D., and G. R. Walter, Prediction of flow and hydraulic head for vertical circulation wells, Ground Water, 30(5), 765-773, 1992.

Rehfeldt, K. R., M. J. Boggs, and L. W. Gelhar, Field study of dispersion in a heterogeneous aquifer, 3, Geostatistical analysis of hydraulic conductivity, Water Resour. Res., 28(12), 3309-3324, 1992.

Sudicky, E. A., A natural gradient experiment of solute transport in a sand aquifer: Spatial variability of hydraulic conductivity and its role in the dispersion process, Water Resour. Res., 22(13), 2069-2082, 1986.

Zlotnik, V., and G. Ledder, Effect of boundary conditions on dipole flow, in Computational Methods in Water Resources $X$, vol. 2, edited by A. Peters et al., pp. 907-914, Kluwer Acad., Norwell, Mass., 1994.

G. Ledder, Department of Mathematics and Statistics, University of Nebraska, Lincoln, NE 68588-0323.

V. Zlotnik, Department of Geology, University of Nebraska, 214 Bessey Hall, P.O. Box 880340, Lincoln, NE 68588-0340.

(Received March 20, 1995; revised August 25, 1995; accepted December 18, 1995.) 